\title{
Comparación de la perspectiva de médicos directivos y generales sobre la actualización en asma
}

\author{
Georgina Eugenia Bazán-Riverón,*凶Jorge Iván Rodríguez-Martínez,* Maricela Osorio-Guzmán,* \\ Laura Evelia Torres-Velázquez, * Javier Sandoval-Navarrete
}

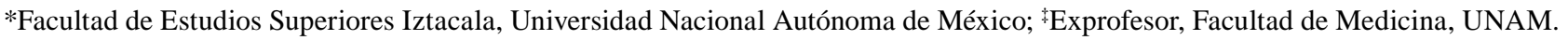
Trabajo recibido: 16-III-2017; aceptado: 19-IX-2017

\begin{abstract}
RESUMEN. Estudios recientes muestran la necesidad de actualizar a los médicos sobre las guías internacionales para el manejo del asma. Se exploraron las variables: educación continua, variables sociales, laborales, administrativas y personales que dificultan su actualización. Participaron 40 médicos (20 directivos y 20 generales); $82.5 \%$ varones y $17.5 \%$ mujeres, con edades de 34-67 años (media = 51.5; DT = 9.5). Se aplicó una entrevista y se analizó mediante la prueba $\chi^{2}$ y el coeficiente kappa. En los resultados se observa que los grupos difieren, pues los directivos consideran que los médicos están limitados por su escaso manejo de inglés e informático; y los médicos consideran problemática la distancia que recorren para actualizarse $\left(\chi^{2}=7.84 ; p<.05\right)$. En las variables administrativas los médicos señalan la deficiente planeación de directivos, el limitado apoyo institucional, mientras que los directivos mencionan la apatía y poco compromiso de los médicos $\left(\chi^{2}=17.477\right.$; $\mathrm{p}<.01$ ). Las coincidencias, ambos grupos reconocen: deficiente conocimiento sobre asma (kappa $=0.93$; IC $=0.95 \%$ : $0.90-1$ ); en el aspecto social y personal consideran que las mujeres están en desventaja para actualizarse (deficiente apoyo institucional y familiar); en las dificultades laborales identifican el exceso de trabajo. Las conclusiones mostraron que se requiere mayor apoyo institucional para la actualización en asma, además del dominio del inglés y de medios informáticos.
\end{abstract}

Palabras clave: Médicos generales, actualización en asma, GINA.

ABSTRACT. Recent studies highlight the need to update physicians on the international guidelines for the management of asthma. Objective was to explore the social, work, administrative and personal variables, as well as continuing education in asthma. Forty physicians took part (20 from medical managements and 20 general-practitioners), $82.5 \%$ males and $17.5 \%$ females, between $34-67$ years of age (average $=51.5$; DT $=9.5$ ), which hinder updating from the aforementioned groups' perspective. Both were interviewed and analyzed by performing the $\chi^{2}$ test and kappa coefficient. Results show differences between the two groups, since managements deem that general-practitioners continuing education is limited due to their poor command of English and computing, whereas general-practitioners consider that the distance they have to travel to update their knowledge is problematic $\left(\chi^{2}=7.84 ; p<.05\right)$; as far as administrative variables are concerned, general-practitioners point out management's poor planning and a lack of institutional support. On another hand, managements complain about general-practitioners disinterest and little commitment $\left(\chi^{2}=17.477 ; p<.01\right)$. Despite these differences, both groups agree on a poor knowledge of asthma $(k a p p a=$ 0.93 ; IC $=0.95 \%: 0.90-1$ ), and, they deem that being a woman is a disadvantage for knowledge updating. Likewise, occupationally speaking, managements and general-practitioners indicated an overload of work. Conclusions show more support is required to improve knowledge of asthma, command of English and computing.

Key words: General practitioners, asthma knowledge updating, GINA.

\section{INTRODUCCIÓN}

La Global Initiative for Asthma (GINA) considera el asma como una enfermedad heterogénea caracterizada usualmente por inflamación crónica, que se define por la historia de síntomas respiratorios tales como silbido, dificultad respiratoria, presión en el pecho y tos que pueden remitir o variar en su intensidad a lo largo del tiempo, con y sin tratamiento, lo anterior aunado a la variable de limitación en el flujo espiratorio de aire. ${ }^{1}$ En
México se calculan 10 millones de pacientes con este padecimiento, de los cuales el $80 \%$ son menores de 5 años. A pesar de que la incidencia de dicha enfermedad varía por región y grupo etario, las proyecciones de la GINA probabilizan en 2025 un aumento a 100 millones de personas con asma a nivel mundial. ${ }^{2}$

En la década de los 90 surge la GINA. Esta iniciativa, además de proponer un protocolo específico de atención para el paciente con asma, identifica al médico general (MG), como un profesional indispensable en su 
manejo. Debido a que él es responsable de la atención médica integral primaria y continua del individuo, funge como vía de referencia y contrarreferencia entre pacientes y médicos especialistas, es responsable de poner en práctica acciones preventivas de salud, además de atender del 80 al $90 \%$ de las enfermedades y los problemas de salud más comunes en la población. ${ }^{3}$ Esta labor es muy compleja debido no sólo a la aparición de nuevas enfermedades, sino por la evolución en la complejidad de trastornos ya existentes, el surgimiento de nuevos conocimientos, técnicas y procedimientos del ejercicio profesional.

Las estadísticas señalan que cada año se descubren alrededor del $10 \%$ de los conocimientos nuevos en el ámbito de la medicina, situación que convierte un $50 \%$ de la formación inicial de los médicos en obsoleta en pocos años (5 aproximadamente), el volumen del conocimiento médico útil se duplica cada 12 o 14 años y se predice que se duplicará en menor tiempo. Por esta razón, es importante que el MG se mantenga siempre en constante actualización a través de diversas estrategias de educación continua (EC), pues la falta de actualización se ve reflejada en el desempeño deficiente de su profesión. ${ }^{4}$

Respecto al tratamiento del asma, desde hace varias décadas se ha intentado difundir la guía GINA, desafortunadamente en diversos estudios se ha comprobado que los esfuerzos no han sido suficientes, ya que la mayoría de los MG y médicos familiares participantes no la conocen. .-9 $^{-9}$

Algunos estudios muestran la necesidad de capacitar a los MG con base en las guías internacionales para el manejo del asma..$^{10-16}$ Asimismo, se han identificado diferentes variables sociales, laborales, administrativas y personales que dificultan su capacitación. ${ }^{17}$ Sin embargo, existe poca información al respecto y menos aun sobre la coincidencia o discrepancia que puede existir entre la percepción de médicos directivos (MD) y la de los propios MG sobre las variables relacionadas con su asistencia a eventos académicos y/o de EC. De tal modo, que el objetivo de esta investigación fue explorar los acuerdos y discrepancias en la perspectiva de los MD y MG sobre las variables que pueden facilitar o dificultar su actualización en asma: conocimientos sobre la guía GINA, características de la EC, aspectos sociales y laborales, variables administrativas y personales.

\section{MATERIAL Y MÉTODO}

Se entrevistó a 40 médicos de la Ciudad de México y del Estado de México que trabajan actualmente en ámbitos públicos y privados; 20 de ellos fueron MD que han cumplido dicha función en promedio 3.8 años, quienes se han desempeñado como coordinadores de EC, directores clínicos y/o directores de Unidades de Medicina Familiar en unidades de primer contacto y en Hospitales Regionales. Los otros 20 participantes fueron MG que nunca han ocupado el cargo de directivos.

El diseño de la investigación fue de corte transversal y descriptiva, los MD fueron seleccionados con un muestreo intencional por informante clave, ${ }^{18-21}$ y los MG con un muestreo de tipo aleatorio en cada ámbito clínico. Se diseñó una cédula de aspectos sociodemográficos (género, edad y estado civil). Los criterios de inclusión fueron el tiempo en el cargo directivo (mínimo dos años) y, en el caso de los MG, que nunca hubieran ejercido un cargo de este tipo y, para ambos grupos, que tuvieran un mínimo de ejercicio profesional de dos años. Se desarrollaron las entrevistas mediante citas con ambos grupos y se asistió a sus consultorios en clínicas públicas y privadas. El instrumento empleado fue una entrevista diseñada y validada mediante un panel de médicos expertos, la cual exploró las variables; actualización y EC, laborales y administrativas; además se revisaron las carpetas testimoniales -las cuales conservan los datos y bitácoras relevantes de las actividades en las unidades clínicas-, para obtener datos fidedignos de factores relacionados con la asistencia a eventos de EC. La entrevista fue realizada de diciembre de 2016 a febrero de 2017 y se basó en tres ejes temáticos (tabla 1).

Previamente a la realización de las entrevistas se mandó el protocolo de investigación al coordinador de EC, al director clínico o al director de la Unidad de Medicina Familiar, una vez aprobado el protocolo se acudió a los consultorios de los médicos participantes, se entregó y recogió el consentimiento informado firmado y se desarrolló la entrevista (sin límite de tiempo). El análisis de resultados se llevó a cabo mediante la clasificación y codificación de las respuestas, que fueron analizados con el programa SPSS versión 19, mediante estadística descriptiva y comparativa para variables de tipo cualitativo (ji cuadrada $\chi^{2}$ ). Asimismo, se aplicó el coeficiente kappa, el cual proporciona la concordancia absoluta en variables de tipo cualitativo, identificando el grado de acuerdo global y aquél que se debe al azar, proporcionando información sobre la fiabilidad entre analistas, observadores o participantes de una investigación. .22-28 $^{2}$

\section{RESULTADOS}

Los resultados se describen de acuerdo con la cédula de datos sociodemográficos, criterios de inclusión y los tres ejes temáticos de la entrevista, mostrando los porcentajes de cada grupo en las respuestas, la 
obtención de $\chi^{2}$ en los casos de la evaluación de diferencias entre categorías de respuestas y en los casos de coincidencias entre las categorías la aplicación de coeficiente kappa. De los 40 médicos el $82.5 \%$ fueron varones y $17.5 \%$ fueron mujeres, con edades de 34 a 67 años (media $=51.5 ; \mathrm{DT}=9.5$ ). Los $20 \mathrm{MD}$ han cumplido dicha función en promedio 3.8 años con un promedio de ejercicio profesional de 25 años (rango $=10-38$; DT $=6.9$ ), se han desempeñado como coordinadores de EC, directores clínicos y/o directores de Unidades de Medicina Familiar en unidades de primer contacto públicas, privadas, y en Hospitales Regionales. Los 20 MG participantes nunca han sido directivos y tienen una media de ejercicio profesional de 26 años (rango $=12-40 ; \mathrm{DT}=8.4$ ). En relación al estado civil, $100 \%$ informan que su profesión es altamente satisfactoria en lo profesional, no así en el ámbito personal, pues es difícil tener una vida familiar armónica (el $70 \%$ es soltero, divorciado o nunca se ha casado), debido al bajo salario percibido en las instancias públicas, que les obliga a dedicarse a la práctica privada, lo cual les lleva a tener dos o tres empleos, impactando sus relaciones familiares y personales. Del $30 \%$ que dijo tener pareja, el $18 \%$ son casados y el resto sólo tienen relaciones ocasionales.

Respecto a la entrevista, en el Eje 1 que contiene los resultados del rubro del inciso a) conocimiento y definición de la GINA (tabla 2), el 57\% de los partici- pantes de ambos grupos responden correctamente (el $30 \%$ de los MD y $27 \%$ de los $M G$ ), ellos informan que sí conocen la guía GINA y la definen correctamente; sin embargo, no se encuentran coincidencias entre los grupos debido a que cada uno privilegia aspectos diferentes de la guía GINA, otro hallazgo importante es que confunden esta última con la Guía Española para el Manejo del Asma (GEMA) y no reconocen la existencia de los diferentes formatos que van dirigidos a pacientes, familiares o a profesionales. En cuanto al inciso b) sobre los pilares de tratamiento de la guía GINA, la muestra total $85 \%$ ( $41 \%$ de los MD y $44 \%$ de los MG) conocen al menos dos pilares del tratamiento y aunque los que mencionan son correctos éstos son diversos, los MD se centran en aspectos farmacológicos; mientras que los MG identifican aspectos centrados en el tratamiento farmacológico y educación para la salud, sin encontrar coincidencias. Cabe señalar que en ambos grupos se omite el control de los factores de riesgo. En cuanto al inciso c) funciones del MG en relación al manejo del paciente con asma, se observó que en ninguno de los dos grupos pudieron dar cuenta de todas las funciones (diagnóstico, canalización con el especialista, control y seguimiento programado del paciente), sólo el $15 \%$ del grupo de médicos $(8 \%$ directivos y $7 \%$ generales) respondió adecuadamente y se observó concordancia en las respuestas (kappa = 0.93; IC. 0.95\%: 0.90). Respecto al inciso d) sobre

Tabla 1. Ejes temáticos y preguntas de la entrevista realizada a los médicos.

\begin{tabular}{|c|c|}
\hline Eje temático & Preguntas \\
\hline $\begin{array}{l}\text { 1. Conocimiento sobre } \\
\text { asma y la guía GINA }\end{array}$ & $\begin{array}{l}\text { a. ¿Conoce y puede definir la GINA? } \\
\text { b. ¿Cuáles son los pilares de tratamiento de la GINA? } \\
\text { c. ¿Cuáles son las funciones del médico general en el manejo del asma? } \\
\text { d. ¿Qué pruebas diagnósticas tiene disponibles para identificar el asma? } \\
\text { e. ¿Considera que necesita capacitación en asma? } \\
\text { f. ¿Cuáles son los temas prioridad en su capacitación? }\end{array}$ \\
\hline
\end{tabular}

a. ¿Cuáles considera que son los aciertos (en logística, organización, contenidos temáticos) de las estrategias educativas en temas emergentes de la salud?

b. ¿Cuál es la modalidad de enseñanza en cursos de actualización mejor aceptada por los médicos?

c. ¿Qué problemas enfrentan los médicos para asistir a la capacitación o actualización?

2. Factores relacionados d. ¿Qué eficiencia terminal ha tenido de los cursos tomados en los últimos 5 años -de manera a la EC de los médicos individual y en los departamentos que los directivos manejan-?

e. ¿Cuáles son los motivos que tienen los médicos para no tomar los cursos de actualización?

f. ¿Cuáles son los motivos que tiene para no asistir a los congresos especializados?

g. ¿Qué soluciones sugiere a los problemas de actualización y capacitación?
3. Manejo de equipo informático
a. ¿Qué equipo informático manejan los médicos?
por el médico general
b. ¿Qué equipo informático emplean en su práctica profesional?
c. ¿Qué uso da a los medios informáticos que tiene? 
Tabla 2. Conocimiento sobre asma y la guía GINA.

\begin{tabular}{|c|c|c|c|}
\hline Preguntas & Directivos & Generales & Kappa \\
\hline a. ¿Conoce y define la guía & Sí & Sí & \\
\hline GINA? & $30 \%$ & $27 \%$ & 0.3 ; IC 95\%: $-0.53-0.63$ \\
\hline b. ¿Conoce los pilares de & Sí & Sí & $0.2 ;$ IC $95 \%:-0.47-0.68$ \\
\hline tratamiento de la GINA? & $41 \%$ & $44 \%$ & \\
\hline $\begin{array}{l}\text { c. ¿Conoce las funciones } \\
\text { del médico general en el } \\
\text { manejo del paciente con } \\
\text { asma? }\end{array}$ & $\begin{array}{l}\text { Sí } \\
8 \%\end{array}$ & $\begin{array}{l}\text { Sí } \\
7 \%\end{array}$ & 0.93; IC 95\%: 0.90-1 \\
\hline $\begin{array}{l}\text { d. Pruebas diagnósticas: } \\
\text { ¿usa espirómetro y/o } \\
\text { flujómetro? }\end{array}$ & $\begin{array}{c}100 \% \text { cree que no es } \\
\text { indispensable el flujómetro } \\
\text { o espirómetro }\end{array}$ & $\begin{array}{c}100 \% \text { cree que no es } \\
\text { indispensable el flujómetro } \\
\text { o espirómetro }\end{array}$ & 1; IC 95\%: 0-1 \\
\hline e. Capacitación & $\begin{array}{l}100 \% \text { derecho de los } \\
\text { médicos }\end{array}$ & $\begin{array}{l}\text { 100\% derecho de los } \\
\text { médicos }\end{array}$ & 1: IC 95\%; 0-1 \\
\hline $\begin{array}{l}\text { f. ¿En qué temas desea ser } \\
\text { capacitado? }\end{array}$ & $\begin{array}{l}100 \% \text { asma, EPOC } \\
\text { e insuficiencia renal }\end{array}$ & $\begin{array}{l}100 \% \text { asma, EPOC } \\
\text { e insuficiencia renal }\end{array}$ & 1: IC 95\%; 0-1 \\
\hline
\end{tabular}

Tabla 3. Distribución de porcentajes de factores relacionados con la educación continua (EC) y diferencias significativas mediante la $\chi^{2}$.

\begin{tabular}{|c|c|c|c|}
\hline \multirow[b]{2}{*}{ Preguntas } & \multicolumn{2}{|c|}{ Médicos } & \multirow[b]{2}{*}{$\chi^{2}$} \\
\hline & Directivos & Generales & \\
\hline $\begin{array}{l}\text { a. ¿Cuáles son los aciertos en } \\
\text { las estrategias educativas en } \\
\text { el área de la salud? }\end{array}$ & $\begin{array}{l}100 \% \text { consideran que la educación } \\
\text { es un derecho médico y obligación } \\
\text { institucional }\end{array}$ & $\begin{array}{l}100 \% \text { consideran que la educación } \\
\text { es un derecho médico y obligación } \\
\text { institucional }\end{array}$ & * \\
\hline $\begin{array}{l}\text { b. ¿Cuál es la modalidad de } \\
\text { enseñanza predilecta? }\end{array}$ & $100 \%$ prefieren cursos presenciales & $100 \%$ prefieren cursos presenciales & * \\
\hline $\begin{array}{l}\text { c. ¿Qué problemas tienen para } \\
\text { asistir a cursos? }\end{array}$ & $\begin{array}{l}\text { El } 100 \% \text { cree que los MG tienen } \\
\text { deficiencias en habilidades digitales } \\
\text { y el manejo del idioma inglés } \\
\text { Sólo directivos }\end{array}$ & $\begin{array}{l}\text { El } 100 \% \text { informa las grandes distan- } \\
\text { cias que deben recorrer }\end{array}$ & * \\
\hline $\begin{array}{l}\text { d. ¿Cuál es la eficiencia terminal } \\
\text { en los cursos? }\end{array}$ & \multicolumn{2}{|c|}{$\begin{array}{l}\text { Eficiencia terminal del } 80 \% \text { (terminan en menos de } 6 \text { meses } 23 \% \text { y el } 57 \% \\
\text { en tiempo y forma) } \\
\text { Abandonan } 20 \%\end{array}$} & ** \\
\hline $\begin{array}{l}\text { e. ¿Qué motivos tiene para no } \\
\text { tomar los cursos de actualiza- } \\
\text { ción? }\end{array}$ & $\begin{array}{l}\text { Directivos } \\
100 \% \text { exceso de trabajo } \\
\text { El } 70 \% \text { sí hay apoyo, pero los MG } \\
\text { no se apoyan entre sí para cubrir la } \\
\text { consulta para actualizarse } \\
\text { El } 100 \% \text { por la distancia que deben } \\
\text { recorrer }\end{array}$ & $\begin{array}{l}\text { Generales } \\
100 \% \text { creen que deben contar con el } \\
\text { apoyo institucional para asistir } \\
\text { El } 16 \% \text { no se sienten apoyados (son } \\
\text { presionados para actualizarse en sus } \\
\text { periodos vacacionales) } \\
\text { El } 100 \% \text { por la distancia que deben } \\
\text { recorrer }\end{array}$ & $p=0.03$ \\
\hline $\begin{array}{l}\text { f. ¿Cuáles son los motivos que } \\
\text { tiene para no asistir a los con- } \\
\text { gresos especializados? }\end{array}$ & $\begin{array}{l}\text { Directivos } \\
\text { El } 76 \% \text { cree que se debe disminuir } \\
\text { apatía en MG } \\
\text { El } 24 \% \text { mejorar compromiso de la } \\
\text { dependencia }\end{array}$ & $\begin{array}{l}\text { Generales } \\
\text { El } 26 \% \text { de los MD cree que disminu- } \\
\text { yen la apatía (mejorando el apoyo } \\
\text { (laboral y económico) } \\
\text { El } 84 \% \text { de los médicos mejorar com- } \\
\text { promiso de la dependencia }\end{array}$ & $\mathrm{p}=.002$ \\
\hline $\begin{array}{l}\text { g. ¿Qué soluciones sugiere a los } \\
\text { problemas de actualización y } \\
\text { capacitación? }\end{array}$ & $\begin{array}{l}\text { Directivos } \\
\text { El } 100 \% \text { no ven solución posible } \\
\text { se requiere un cambio de actitud y } \\
\text { más recursos humanos }\end{array}$ & $\begin{array}{l}\text { Generales } \\
\text { El } 100 \% \text { cree que no hay solución. } \\
\text { Se necesita cambio administrativo y } \\
\text { económico }\end{array}$ & * \\
\hline
\end{tabular}

* No es posible aplicar la $\chi^{2}$ en el programa estadístico SPSS, por tener celdas con valor igual a 0.

${ }^{* *}$ No se aplica la $\chi^{2}$ debido a que sólo se recoge la información del grupo de MD. 
las pruebas diagnósticas, el $100 \%$ de ambos grupos dijeron sólo tener a su alcance pruebas de gabinete y consideran que «no es indispensable» ni el flujómetro ni el espirómetro. En cuanto al inciso e), el cual corresponde a la capacitación, el $100 \%$ en ambos grupos piensan que es un derecho de los médicos y una obligación de la institución, la cual debería proporcionar apoyo económico, así como la cobertura de la consulta cuando el médico se ausente para actualizarse, asimismo, sugieren aprovechar las sesiones médicas semanales para capacitación. Llama la atención que ninguno de los dos grupos ve como opción los cursos no presenciales ni las plataformas digitales de enseñanza. Respecto a los temas prioritarios para actualización (inciso f), ambos grupos coinciden en un $100 \%$ refiriendo la urgente necesidad de capacitarse y actualizarse, no sólo en asma, sino en manejo del paciente con EPOC y la atención integral del paciente renal, lo cual coincide con diferentes fuentes que identifican estos padecimientos con una incidencia creciente en México. ${ }^{29,30}$

Sobre el Eje 2 de la entrevista, que considera factores relacionados a la EC de los médicos (tabla 3), se trataron los aciertos de las estrategias educativas en el área de la salud -inciso a)-en este rubro, los MG y MD coinciden en un $100 \%$ en que los aciertos son considerar la capacitación de los médicos como un derecho del médico y una obligación institucional. Respecto al inciso b) relacionado con las modalidades de enseñanza mejor aceptadas por los médicos, ambos grupos en un $100 \%$ están de acuerdo en que los cursos predilectos son los presenciales y en que deben contar con el apoyo institucional para asistir, no obstante, difieren respecto a las modalidades de enseñanza, $100 \%$ de los MD afirman que los MG no se inscriben a las plataformas educativas por desconocimiento en el manejo de medios informáticos y por su limitado manejo del idioma inglés; por su parte el $100 \%$ de los MG sólo consideran los cursos presenciales, los que son a distancia no representan una opción viable para ellos. Esto último se relaciona con el inciso c) sobre los problemas para asistir a los cursos de actualización, pues los MG afirman que su principal problema es la distancia que deben recorren en los traslados. Respecto a la eficiencia terminal de los cursos tomados en los últimos 5 años correspondiente al inciso d), los MD mediante las carpetas testimoniales mostraron que terminan los cursos el $80 \%$; de este porcentaje un $25 \%$ abandona y retoma -en promedio un año más tarde- y el $20 \%$ abandonan los cursos y no los terminan.

En relación con los problemas que enfrentan los médicos para asistir a los cursos de actualización -incisos e) y f)-, las diferencias fueron significativas $\left(\chi^{2}=7.84\right.$; $p<0.05)$, pues los MD aluden que si se les apoya -se pueden ausentar-pero entre los MG no se apoyan para cubrir la consulta cuando acuden a actualizarse y sugieren que la causa es la apatía de los MG; sin embargo, los MG no se sienten apoyados, refirieron prácticas comunes realizadas por los MD, quienes les presionan para que asistan a actualizarse en sus períodos vacacionales, esperando que asistan a eventos académicos especializados y congresos sin proporcionar apoyo económico $\left(\chi^{2}=17.477 ; p<.01\right)$. En este mismo inciso ambos grupos están de acuerdo en un $100 \%$ en que el problema mayor es el exceso de trabajo.

Es importante señalar que sobre los incisos e) y f), todos los entrevistados señalaron que se observa un fenómeno de inequidad de género, coincidiendo en que las mujeres viven una situación de discriminación, hecho que ejemplifican con una práctica habitual, la cual consiste en ofrecer las oportunidades a los varones, pues ellos no tienen que «pedir permiso» a sus parejas. También informan que se toman estas medidas, pues en el caso de los médicos varones ni sus familias, ni sus hijos «son un obstáculo» para asistir, como lo son para las mujeres. Las soluciones que refiere el inciso g), no tuvieron acuerdo de ningún tipo, pues los MD, creen que los MG deben ser «menos apáticos» y «más comprometidos» con su dependencia; y los MG refieren que las dependencias y los MD para evitar la apatía en los MG deberían tener mejores estrategias organizativas y de apoyo, tanto administrativas para reducir la carga de consulta mientras se actualizan, como económicas para que pudieran asistir a eventos académicos -congresos especializados, simposios, etcétera-. Asimismo, respecto al inciso g), las soluciones que sugieren los médicos a los problemas de actualización y capacitación, afirman que se debe acabar con la discriminación que se hace a los médicos mexicanos, prefiriendo siempre traer ponentes extranjeros (lo cual genera un gasto excesivo), aun cuando los médicos mexicanos tengan la preparación adecuada para impartir los cursos de actualización o capacitación.

En cuanto al Eje 3 de la entrevista (tabla 4), relacionado al manejo de equipo y las tecnologías de la información y la comunicación (TIC), se identificó que el $100 \%$ de los médicos maneja el sistema de cómputo de su PC institucional (para enviar correos electrónicos, elaborar recetas, realizar notas al expediente del paciente), también que el $100 \%$ de ambos grupos usan lap top, o algún aparato inteligente (teléfono o Tablet); no obstante, el uso se limita a redes sociales, búsquedas inmediatas sobre posologías de medicamentos y novedades de los laboratorios clínicos o farmacológicos. Lo cual confirma los incisos a), b) y c) del tercer eje y las observaciones de los MD sobre la subutilización de los recursos informáticos, tanto institucionales como personales. 
Tabla 4. Distribución de porcentajes en el manejo de equipo informático por el médico general.

\begin{tabular}{lll}
\hline & & \multicolumn{2}{c}{ Médicos } \\
\cline { 2 - 3 } Preguntas & Directivos & Generales \\
\hline a. ¿Qué equipo informático manejan & $100 \%$ manejo & $100 \%$ manejo \\
los médicos? & PC institucional & PC institucional \\
& Lap top, teléfono inteligente y tablet & Lap top, teléfono inteligente y tablet \\
b. ¿Qué equipo informático emplean & $100 \%$ emplean & $100 \%$ emplean \\
en su práctica profesional? & PC institucional & PC institucional \\
& Lap top, teléfono inteligente y tablet & Lap top, teléfono inteligente y tablet \\
c. ¿Qué uso da a los medios informá- & PC: enviar correo electrónico, elaborar & PC: Enviar correos electrónicos, \\
ticos que tiene? & recetas, expedientes y notas médicas, & elaborar recetas, expedientes y notas \\
& Teléfono inteligente Lap top y tablet: & médicas Teléfono inteligente Lap top y \\
& para redes sociales, posologías, & tablet: para redes sociales, posologías, \\
& navegar por Internet & navegar por Internet \\
\hline
\end{tabular}

\section{DISCUSIÓN}

A pesar de que la guía GINA surge en la década de los 90 y su página Web tiene sitios de divulgación, pocos médicos la conocen y pueden dar cuenta de sus innovaciones ${ }^{31}$ como se comprobó en el eje uno de la entrevista y con los porcentajes de eficiencia terminal, los cuales, son mejorables. En el eje dos, se encontró la preferencia por cursos presenciales; y como una de sus limitantes principales, el que asistir les obliga a recorrer grandes distancias. Así que se debe favorecer la consideración y aceptación de la EC mediante la inclusión de las TIC. De esta manera, una EC exitosa en esta población debe contener los dos objetivos básicos mencionados por Narro en 2004, que reúnen las opiniones de los encuestados en esta investigación: por un lado, se debe formar a los MG en habilidades digitales fundamentales para que puedan favorecerse con los avances tecnológicos y, por el otro lado, mejorar los planes estratégicos de la administración de salud que tiene que ver con brindar las mejores condiciones laborales a estos profesionales. ${ }^{3}$ Esto permitirá satisfacer la necesidad de actualización que de manera consistente se ha detectado en la literatura respecto a asma y sobre cualquiera de los padecimientos que los MG citen como prioritarios para actualizarse. ${ }^{10-15,32-34}$ De este modo, proporcionar las condiciones para la actualización de los $M G$ mediante programas de EC fortalecerá la práctica de estos profesionales y la visión holística del paciente que, si bien no es nueva, sí es un aspecto que se ha privilegiado en las guías internacionales para el manejo de enfermedades como el asma, ${ }^{1,31}$ EPOC $^{35}$ e insuficiencia renal; ${ }^{36}$ las cuales, ellos han seleccionado para ser capacitados por la elevada frecuencia con que se presentan en su consulta.
Con el eje dos de la entrevista, también se detectaron las limitaciones de estos profesionales por el exceso de trabajo y la falta de tiempo debido al colapso de los sistemas de salud, el cual se debe al excesivo aumento de las enfermedades crónicas que cada vez más se asocian a estilos de vida mórbidos (vida sedentaria, mala nutrición, hábitos nocivos como el uso de alcohol y tabaco, entre otros) que tienen que ver con los factores que alude Lifshitz en 2016 como causantes de los «fracasos de la medicina». ${ }^{37} \mathrm{El}$ autor hace una interesante reflexión sobre la práctica de la medicina reconsiderando el papel del médico en la salud y buscando un cambio trascendente de una medicina «medicalizada y paternalista» a una medicina preventiva que logre educar al paciente y generar en él un cambio de actitud transformándolo de un paciente pasivo a uno proactivo y responsable de su salud, hecho que da paso a la visión multidisciplinaria que plantea un tratamiento conjunto que puede ser liderado por el MG, quien tiene el perfil profesiográfico para hacerlo como lo indica Narro en $2004^{3}$ y como se corrobora en este estudio, pues el MG suele privilegiar en sus intervenciones la educación para la salud, a diferencia de los MD quienes se centran más en aspectos farmacológicos.

La percepción de los MG y MD sobre las problemáticas relacionadas a su actualización profesional son consensuadas en ambos grupos cuando se refieren a la preferencia de los métodos de enseñanza presenciales; y a las condiciones laborales que tienen (exceso de trabajo, inequidad de género y pocos apoyos institucionales). Sin embargo, existen importantes diferencias como la visión de los MD sobre la apatía, la falta de compromiso de los MG para actualizarse en medios informáticos y el manejo del idioma inglés. ${ }^{15,16}$ Por otra parte, la visión de los MG señala la falta de apoyos de su institución para asistir a cursos de actua- 
lización, pues requieren desplazarse o ausentarse de su actividad cotidiana y esto no es permitido. Ambas perspectivas diferentes confluyen en un punto, el desconocimiento de las plataformas educativas, pues la mayoría de éstas son actividades a distancia y tienen versiones en español. Además, por su propia naturaleza permiten que el profesional acceda en los horarios que le sean convenientes desde dispositivos remotos (teléfono inteligente, Tablet o computadoras), sin tener que trasladarse.

Por tanto, los retos más inmediatos tienen que ver con dar a conocer el funcionamiento de plataformas de EC a distancia, vencer la resistencia de las generaciones con mayor edad de MG para el empleo de medios informáticos y generar mecanismos de acceso a la educación a distancia como primera elección brindándoles las habilidades necesarias. ${ }^{33-35}$ Eliminar el rezago digital, también se vincula con las temáticas que refieren para ser capacitados, además del asma, consideran urgente, recibir actualización sobre EPOC e insuficiencia renal; no obstante, deben considerarse los resultados obtenidos en este estudio para poder garantizar que los avances de las guías internacionales sobre dichos padecimientos publicadas anualmente puedan ser accesibles a la población de MG, pues de continuar con el rezago digital, aun cuando cada vez se difunden más los avances de la medicina éstos no están al alcance de estos profesionales.

Partiendo de los resultados obtenidos se corrobora la ineficacia de algunos métodos actuales para difundir protocolos de atención como la guía GINA. Este hecho debe conducir a estrategias precurrentes dirigidas a proporcionar a los médicos las habilidades necesarias para que incorporen a sus destrezas, el manejo de las nuevas plataformas de enseñanza.

Ambos grupos de médicos argumentan que los mayores de 50 años no están «suficientemente capacitados», para ingresar a plataformas educativas, mostrando abiertamente una gran resistencia a actualizarse por estos medios. La brecha digital que se abre entre los médicos de mayor edad y los médicos más jóvenes es muy amplia, se ha convertido en todo un reto dentro de las instituciones, pues los médicos de este estudio de ambos grupos refieren que tienen dificultades para seguir los acelerados avances informáticos y consideran que las nuevas tecnologías, están «lejos» de su alcance; así que con ellos, debe partirse desde un proceso de sensibilización, motivación, información y capacitación para el uso de nuevas tecnologías.

En este sentido, las intervenciones que se planteen para resolver esta compleja situación tendrán que considerar la resistencia de estos profesionales al empleo de las TIC, su limitado dominio del idioma inglés y la urgente intervención de la perspectiva de género en este ámbito profesional para hacer que las mujeres dejen de ser discriminadas para recibir apoyo y para tener acceso a la actualización y a la EC.

Finalmente, el problema de actualización y asistencia a eventos académicos como congresos y simposios es un reto aparentemente fácil de superar, pues actualmente las plataformas educativas son cada vez más sencillas. Asimismo, la posibilidad de asistir virtualmente a eventos actuales y de gran calidad académica puede verse superada mediante el empleo de videoconferencias, foros de debate y las diferentes actividades académicas a distancia que promueven PRONADAMEG y CONAMEGE que están avalados por la Facultad de Medicina de la UNAM. Lo anterior puede verse favorecido, ya que como se comprobó en el eje tres de la entrevista, los médicos poseen los dispositivos necesarios para acceder a estas tecnologías, con lo cual sólo se debe instigar y capacitar para su uso y que teléfonos inteligentes, tabletas y computadoras portátiles no se vean subutilizadas.

\section{REFERENCIAS}

1. Global Initiative for Asthma (GINA). Guía de bolsillo para el manejo y la prevención del asma 2015. Fecha de consulta: 01-I-2017. Disponible en: http://www. ginasthma.org/local/uploads/files/GINA_Pocket_ Spanish2014.pdf

2. Huerta LJG, Jiménez GC, Gómez GDR, Travera RMG, López AJM. Evaluación de la calidad clínica y metodológica de las guías de práctica clínica para el manejo del asma en pacientes pediátricos. Alerg Asma Inmunol Pediatr 2011;20(1):5-28.

3. Narro-Robles J, Ruiz-Ruisánchez A. El papel del médico general en la atención médica en el México actual. Gac Med Méx 2004;140(Supl 1):S13-S20.

4. Karchmer KS. Educación médica continuada. Necesidades de proyección. Acta Med 2006;4(1):61-66. Disponible en: http://www.medigraphic.com/pdfs/actmed/am2006/am061k.pdf

5. Segura MN, Del Rivero L, Olvera J, et al. El uso de las Guías Internacionales de Diagnóstico y Tratamiento del Asma (GINA) en la práctica clínica de los médicos familiares. Rev Alerg Mex 2001;48(6):159-162.

6. Segura MN, Barragán EML, Guido BR, et al. La iniciativa global para el asma (GINA) y los médicos residentes de medicina familiar. Impacto de una estrategia educativa. Rev Alerg Mex 2005;52(1):3-6.

7. Ruiz EJ, González DSN, Galindo RG, Arias CA, Canseco VC, Valdez RMA. Evaluación de un curso de educación en asma para médicos del primer nivel de atención. Rev Alergia Méx 2005;52(2):83-89.

8. Baeza MA, Rebolledo CA. Elaboración de un cuestionario para evaluar el conocimiento sobre el 
asma infantil en estudiantes de medicina. Rev Alergia Méx 1998;45(2):49-53.

9. Plaza V, Bolívar I, Giner J, et al. Knowledge of and attitudes and adherence to the Spanish Guidelines for Asthma Management (GEMA) among Spanish health care professionals: the GEMA test Project. Arch Bronconeumol 2008;44(5):245-451.

10. Rodríguez MJI, Bazán RGE, Paredes RMP, Osorio GM, Caso MA, Sandoval NJ. Evaluación del conocimiento de GINA en médicos generales y especialistas del estado de Puebla (México). Alerg Asma Inmunol Pediatr 2004;13(3):94-98.

11. Becerril ÁM, León AF, Ángeles GU. Evaluación del conocimiento de la GINA en médicos de diferentes niveles de atención. Rev Alergia Méx 2007;54(2):29-33.

12. Zavala AJA, Furuya MEY, Vargas MH. Competencia clínica de médicos de urgencias en la atención de niños con exacerbación asmática. Rev Alergia Méx 2008;55(4):139-147.

13. Bazán RGE, Torres VLE, Osorio GM, Reyes LAG, Garrido GG. ¿Qué saben los médicos generales sobre métodos diagnósticos en el control del asma? En: Orozco GM, Caballero VIK, editores. Psicología Latinoamericana: experiencias, desafíos y sus compromisos sociales. México: Asociación Mexicana de Alternativas en Psicología; 2014. p. 341-349. Disponible en: http:// www.alfepsi.org/attachments/article/348/LIBRO\%20 Psicolog\%C3\%ADa\%20 Latinoamericana_\%20 Experiencias, $\% 20$ desaf $\%$ C $3 \%$ ADos $\% 20 \bar{y} \% 20$ compromisos\%20sociales.pdf

14. Sánchez SC, Bazán RGE, Torres VLE, Osorio GM, Garrido GA, Reyes LGA. Validación de un instrumento para evaluar el conocimiento de médicos generales sobre la guía internacional en el manejo del asma GINA: informe preliminar. Rev Electr Psic Iztacala 2014;18(3):1103-1118. Disponible en: http://www.journals.unam.mx/index. php/repi/article/view/52651

15. Bazán RG, Rodríguez MJ, Torres VL, Osorio GM, Sandoval N. Educación continua (EC): Conocimiento de los médicos generales sobre la Guía Internacional para el Manejo del Asma. Rev Electr Psic Iztacala 2015;18(4):1328-1345.

16. Bazán RGE, Rodríguez MJI, Sandoval NJ, Sánchez SC, Bautista GYM. Conocimiento de los médicos sobre la guía (GINA) para el manejo del asma: datos preliminares. En: Orozco GM, Caballero VIK, editores. Psicología Latinoamericana: experiencias, desafíos y sus compromisos sociales. México: Asociación Mexicana de Alternativas en Psicología; 2014. p. 351-364. Disponible en: http://www.alfepsi.org/attachments/article/348/ LIBRO\%20Psicolog\%C3\%ADa\%20Latinoamericana_\%20 Experiencias, $\% 20$ desaf $\%$ C $3 \%$ AD os $\% 20$ y $\% 20$ compromisos\%20sociales.pdf

17. Fernández PJA. Educación médica continua y desarrollo de una profesión. La percepción de los actores. Rev Educac Desarrollo 2014;(28):21-35.

18. Moraima CM, Auxiliadora ML. El análisis de contenido: Una forma de abordaje metodológico. Laurus [Internet]. 2008;14(27):129-144. Disponible en: http://www. redalyc.org/articulo.oa?id $=76111892008$. Fecha de consulta: 18 de agosto de 2017.

19. Robledo MJ. Observación participante: informantes claves y rol del investigador. Nure Investigación 2009;(42):1-4. Disponible en: file:///C:/Users/HP/ Downloads/informante_clave2.pdf

20. Murillo FJ, Martínez-Garrido C. Investigación etnográfica. Métodos de Investigación educativa en educación especial. 3ra. ed. 2010; Madrid: UAM. p. 1-15. Disponible en: https://www.uam.es/personal_pdi/ stmaria/jmurillo/InvestigacionEE/Presentaciones/ Curso_10/I_Etnografica_Trabajo.pdf

21. Rodríguez GG, Gil FJ, García JE. Metodología de la investigación cualitativa. Granada, España: Ediciones Aljibe; 1996. Disponible en: https://media. utp.edu.co/centro-gestion-ambiental/archivos/.../ investigacioncualitativa.doc

22. Fleiss JL. Measuring nominal scale agreement among many raters. Psychol Bull 1971;76(5):378-382.

23. Fleiss JL, Cohen J, Everitt BS. Large sample standard errors of kappa and weighted kappa. Psychol Bull 1969;72:323-327.

24. Sim J, Wright CC. The kappa statistic in reliability studies: use, interpretation, and sample size requirements. Phys Ther 2005;85(3):257-268.

25. López UGI, Pita FS. Investigación: Medidas de concordancia: el índice de Kappa. Cad Aten Primaria 1999;6:169-171. Fecha de consulta: 20-IV-2017. Disponible en: www.fisterra.com

26. Bermejo FB. Fiabilidad. Calidad de la información. En: Bermejo FB, editor. Epidemiología clínica aplicada a la toma de decisiones en medicina. Monografía 1. Ciencias básicas. España: Anales del Sistema Sanitario de Navarra; 2001. p. 53-67.

27. Abraira V. El índice kappa. SEMERGEN 2000;27:247-249. doi: 10.1016/S1138-3593(01)73955-X

28. Piloto MM. Estadística piloto: paquete estadístico digital educacional para las investigaciones epidemiológicas. Rev Ciencias Médicas 2010;14(4). Fecha de acceso: 01II-2017. Disponible en: http://publicaciones.pri.sld.cu/ index.php/publicaciones/article/view/709

29. OMS. Personal sanitario. Organización Mundial de la Salud. Fecha de acceso: 20 de mayo, 2015. Accesible en: URL: http://www.who.int/hrh/workforce_(MD)gs/es/

30. OECD. Estadísticas de la OCDE sobre la salud 2014, México en comparación. OECD. Fecha de acceso: 20 de mayo, 2015. Accesible en: URL: http://www.oecd.org/els/healthsystems/Briefing-Note-MEXICO-2014-in-Spanish.pdf

31. Bautista GYM, Bazán RGE, Torres VLE, Osorio GM, Garrido GA, Reyes LGA. Percepción de los médicos generales sobre el trabajo multidisciplinario en la atención del paciente con asma. Rev Electr Psic Iztacala 2016;19(1):115-130. Fecha de acceso: 04-III-2017. Disponible en: http://www.revistas.unam.mx/index. php/repi/article/view/54895/48762

32. Ablan CF, Grüber BE, Arocha RJI. Estrategias y desarrollo para la educación médica continúa a distancia. Difusión de la información. Rev Venez Endocrinol Metab 2012;10(Supl 1):143-145. 
33. Orozco ROA, Guillén CDM, Martínez VAL. Dispositivos móviles como una nueva alternativa de enseñanza en la práctica clínica de la enfermería. Rev CONAMED 2014;19(4):166-171.

34. Gracia-Ramírez A, Urrusti-Sanz J, de la Llata-Romero M. Perfil del médico inscrito en el Programa Nacional de Actualización y Desarrollo Académico para el Médico General. Gac Med Mex 2004;140 Supl 1:S59-S62.

35. La Global Initiative for Chronic Obstructive Lung Disease (GOLD). Guía de bolsillo para el diagnóstico, manejo y prevención de la EPOC. Una Guía para profesionales de la asistencia sanitaria. 2017. Fecha de acceso: 04-III-2017. Accesible en: http://goldcopd.org/wpcontent/uploads/2016/04/wms-spanish-Pocket-GuideGOLD-2017.pdf

36. ISN-SLANH. Programs Supporters. International Society of Nephrology (ISN). Sociedad Latinoamericana de Nefrología e Hipertensión (SLANH). 2017. Fecha de acceso: 23-IV-2017. Accesible en: https://www.theisn. org/programs/programs-supporters

37. Lifshitz A. Los fracasos de la medicina. Gac Med Mex 2016;152(3):293-294.

\section{$\triangle$ Correspondencia:}

Dra. Georgina Eugenia Bazán-Riverón Unidad de Investigación Interdisciplinaria en Ciencias de la Salud y la Educación (UIICSE), 1er piso Cub. 6. Facultad de Estudios Superiores Iztacala, UNAM.

Av. de los Barrios Núm. 1, colonia Los Reyes Iztacala, 54090, Tlalnepantla, Edo. de México. Correo electrónico: gebrm@yahoo.com.mx

Los autores declaran no tener conflicto de intereses. 